\title{
Joint Learning for Emotion Classification and Emotion Cause Detection
}

\author{
Ying Chen ${ }^{1}$, Wenjun Hou ${ }^{1}$, Xiyao Cheng ${ }^{1}$, Shoushan $\mathbf{L i}^{2}$ \\ ${ }^{1}$ College of Information and Electrical Engineering, China Agricultural University, China \\ ${ }^{2}$ Natural Language Processing Lab, Soochow University, China \\ \{chenying, houwenjun, chengxiyao\} @cau.edu.cn \\ lishoushand suda.edu.cn
}

\begin{abstract}
We present a neural network-based joint approach for emotion classification and emotion cause detection, which attempts to capture mutual benefits across the two sub-tasks of emotion analysis. Considering that emotion classification and emotion cause detection need different kinds of features (affective and event-based separately), we propose a joint encoder which uses a unified framework to extract features for both sub-tasks and a joint model trainer which simultaneously learns two models for the two sub-tasks separately. Our experiments on Chinese microblogs show that the joint approach is very promising.
\end{abstract}

\section{Introduction}

The analysis of emotions in texts is an important task in NLP. Traditional studies treat this task as a pipeline of two separated sub-tasks: emotion classification and emotion cause detection. The former identifies the category of an emotion and the latter detects the cause of an emotion. This separated framework makes each sub-task more flexible to deal with, but it neglects the relevance between the two sub-tasks. In this paper, we explore joint approaches which can capture mutual benefits across the relevant two sub-tasks. To the best of our knowledge, this work is the first attempt to incorporate both emotion classification and emotion cause detection into a unified framework.

Although emotion classification relies on affective features and emotion cause detection needs event-based features, we propose a joint encoder which uses a unified framework to ex- tract features for both emotion classification instances and emotion cause detection instances. Then, we propose a joint model trainer which simultaneously learns two models for the two sub-tasks separately. The experiments on Chinese microblogs show that our joint approach can effectively learn models for both sub-tasks.

\section{Our Approach}

\subsection{Corpus}

In this paper, we use the human-labeled emotion corpus provided by Cheng et al. (2017) as our experimental data (namely Cheng emotion corpus). To better explain our work, we adopt twitter's terminology used in Cheng et al. (2017). Cheng emotion corpus can be considered as a collection of subtweets. For each emotion in a subtweet, all emotion keywords expressing the emotion are selected, and then the class and the cause of the emotion are annotated. The emotion categorization used in Huang et al. (2016) is adopted, which includes four basic emotions (i.e., joy, angry, sad and fearful) and three complex emotions (i.e., positive, neutral and negative). E.g. in the following example, the class of the emotion keyword ("ن)") is $s a d$, and the cause of the emotion is "only I was at home again".

Chinese : 兴冲冲勒跑回家 发现又是我一个人再

家。。早知道就去蹭饭了

English Translation: I was very excited to run back home. I found that only I was at home again. If I

knew it earlier, I would have a meal for free.

Figure 1: An example of a subtweet 


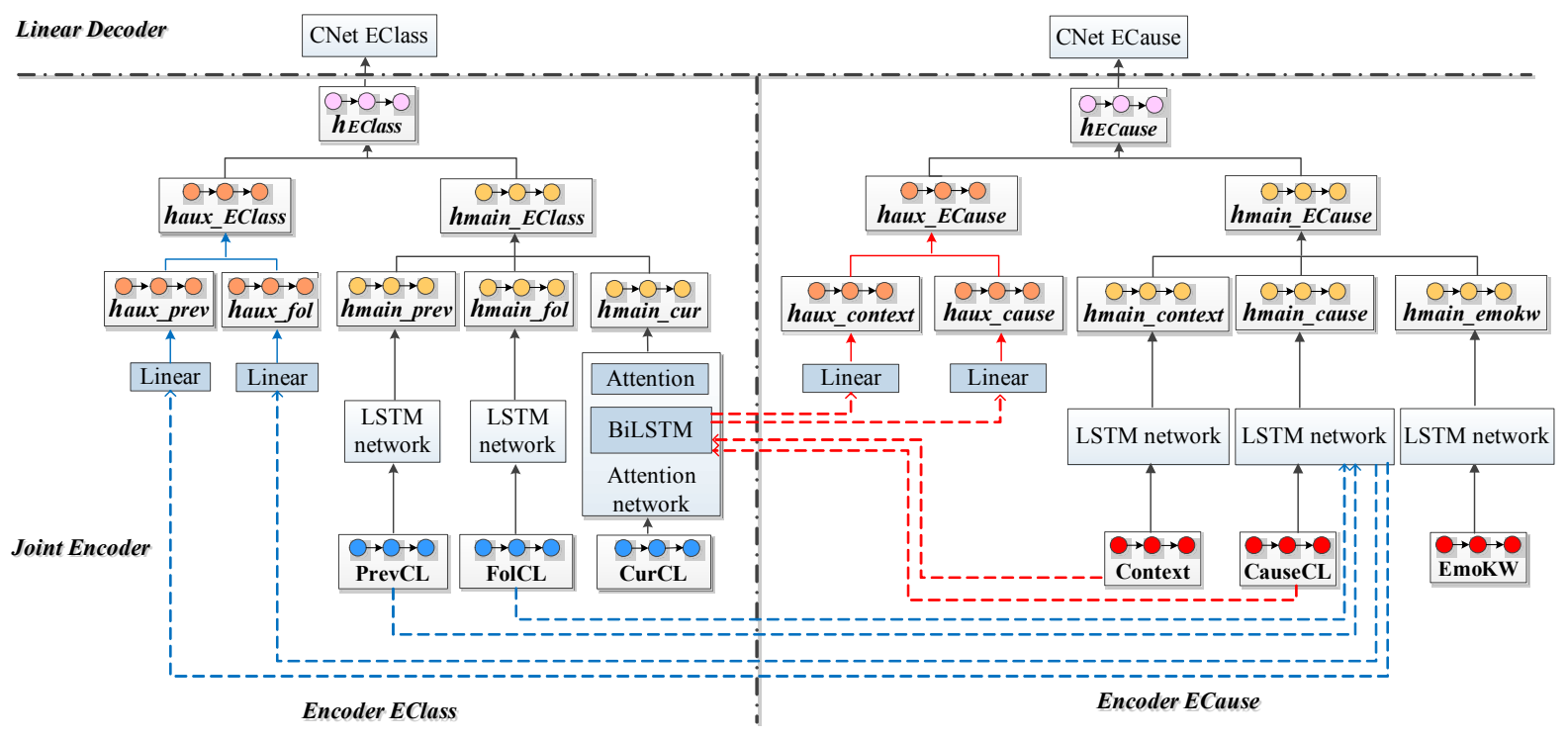

Figure 2: The framework of our joint approach

\subsection{Problem Formulation}

In this paper, both the emotion classification subtask (namely EClass) and the emotion cause detection sub-task (namely ECause) are clauselevel. Given an instance which is a clause in a subtweet, EClass assigns one of seven labels (i.e. six emotion classes and label 'non-emotion' which indicates the absence of an emotion) to the instance. Notice, because of the extremely low percentage of emotion 'fearful' ( $\sim 0.6 \%$ in $\$ 3.1$ Table 1), we ignore this emotion class in EClass. Given an instance which is a pair of $<$ an emotion keyword, a clause in the subtweet>, ECause assigns a binary label to the instance to indicates the presence of a causal relation. Moreover, the clause-level EClass can effectively avoid the problem of multiple emotions ( $\mathrm{Li}$ et al., 2015) because clauses are a kind of fine-grained texts.

Furthermore, the input text of an EClass instance contains three sequences of words: the previous clause (i.e. PrevCL), the current clause (i.e. $\operatorname{CurCL}$ ), and the following clause (i.e. FolCL). The previous clause and the following clause provide contextual information for the current clause. The input text of an ECause instance also has three sequences of words: the emotion keyword (i.e. $E m o K W$ ), the current clause (i.e. Cause CL) and the context between EmoKW and CauseCL. The emotion keyword serves as an anchor, the current clause gives the description of an event which may cause the emotion, and the context provides complemental information for the event. Moreover, each word is represented with a vector from our word embedding model which is trained with word $2 \mathrm{vec}^{1}$ and the tweet corpus of Cheng et al. (2017).

\subsection{The Joint Approach}

As shown in Fig. 2, there are two parts in our joint approach which is based on neural networks: a joint encoder (the lower part) which extracts feature representations for both EClass instances and ECause instances, and a linear decoder (the upper part) which assigns labels to instances according to their representations.

\section{Neural Networks}

In the joint encoder, there are two neural networks (the attention network and the LSTM network), and each neural network is composed of several layers: bidirectional LSTM (i.e. BiLSTM) and attention. The BiLSTM layer focuses on the extraction of sequence features, and the attention layer focuses on the learning of word importance (weights). Because of the feature sparse problem in our small-scaled experimental data, the attention network often cannot effectively extract features to represent an event (see §3.2). Thus, in our joint encoder, we use the attention network to extract affective features (e.g. "하)" in Fig. 1) and the LSTM network to extract event-based features (e.g. "I found that only I was at home again" in Fig. 1).

\footnotetext{
${ }^{1}$ https://code.google.com/p/word2vec/
} 
The attention network: we implement the attention network used in Felbo et al. (2017), which includes two layers: a BiLSTM layer which extracts a sequence feature for each input word, and an attention layer which represents the input sequence using weighted words.

The LSTM network: the network uses a BiLSTM layer to capture a sequence feature for each input word, and then uses the average of those features as the representation of the input sequence.

In the linear decoder, there are two classification networks (CNet EClass and CNet ECause) for EClass and ECause separately. Each classification network uses a linear layer to build a probabilistic classification model.

\section{The Joint Encoder}

As shown in Fig. 2, there are two sub-encoders in our joint encoder: Encoder EClass (the left part) which provides a representation for an EClass instance, and Encoder ECause (the right part) which extracts a representation for an ECause instance. Given an instance, one sub-encoder extracts a main representation (through the black lines in Fig.2) and the other sub-encoder provides an auxiliary representation (through the blue or red lines in Fig.2). Then, the concatenation of the two representations serves as the final representation for the instance (i.e. $h_{\text {EClass }}$ or $h_{\text {ECause }}$ in Fig.2). In order to deal with the case that a main representation may be overwhelmed by its corresponding auxiliary representation, linear layers are used to reduce the dimensions of auxiliary representations. Moreover, there are three sequences of words either in the input text of an EClass instance or in the input text of an ECause instance. In order to effectively use these input sequences, a multi-channel structure is chosen, which encodes the input sequences one by one.

Encoder EClass: given the three sequences of words in an EClass instance (PrevCL, CurCL and FolCL), the attention network is applied to CurCL to extract an affective representation, and the LSTM network is applied to PrevCL and FolCL separately to extract two event-based representations. Then, the concatenation of the three representations is used as the main representation (i.e. $h_{\text {main_EClass }}$ ). Furthermore, in order to extract more contextual information, the LSTM network of Encoder ECause is applied to PrevCL and FolCL (through the blue lines in Fig. 2) to extract the auxiliary representation (i.e. $h_{\text {aux_EClass), which }}$ provides another event-based view for our emotion classification.

Encoder ECause: in order to separately deal with the three sequences of words (EmoKW, CauseCL and Context) in an ECause instance, the LSTM network is applied to each input sequence and then the concatenation of the three representations is used as the main representation (i.e. $h_{\text {main_ECause). Furthermore, for each input sequence }}$ (CauseCL or Context), the BiLSTM layer in the attention network is used to extract more eventbased features (through the red lines in Fig. 2), and those features serve as an auxiliary representation (i.e. $h_{\text {aux_ECause }}$ ) which provides another event-based view for our emotion cause detection.

\section{The Joint Model Trainer}

During training, two models (JMEClass and $J M E C a u s e)$ are learned simultaneously for the two sub-tasks (EClass and ECause) separately. Model JMEClass contains Encoder EClass and CNet EClass, and Model JMECause contains Encoder ECause and CNet ECause. Although each model uses auxiliary representations from the other model, but the learning of the model focuses on its own parameters. In other words, gradient calculation is disabled along the dashed lines in Fig. 2.

In each episode, the batch of input data is composed of two sets of instances: EClass subbatch containing only EClass instances and ECause sub-batch containing only ECause instances. Given the batch of data, the parameters of each model are updated according its corresponding loss function. E.g., Model JMEClass uses only the EClass sub-batch, and its loss function is the mean squared errors of the instances in the sub-batch. In our joint model trainer, the two models are optimized using their own loss functions as pipeline model training does, but they use up-to-date auxiliary representations from each other to help optimization.

\section{Experiments}

\subsection{Experimental Setup}

In Cheng emotion corpus, there are $\sim 3,000$ subtweets, $\sim 11,000$ instances for EClass, and $\sim 13,000$ instances for ECause. Moreover, Table 1 lists the class distribution in Cheng emotion corpus for EClass. All experiments in this paper are 
trained and tested by 5 -fold cross-validation on Cheng emotion corpus, and all the results reported are the average ones of 5-fold cross-validation performances. We use the precision, recall and Fscore as our evaluation metrics. However, because of the high percentage of label 'nonemotion' in EClass (see Table 1) and label ' 0 ' in ECause, similar to previous work ( $\mathrm{Li}$ et al. 2015; Felbo et al., 2017; Cheng et al., 2017; Gui et al., 2017), we report only the evaluation metrics of the six emotion classes for EClass and the evaluation metrics of label 'I' for ECause.

\begin{tabular}{|l|c|l|c|}
\hline Class & $\%$ & Class & $\%$ \\
\hline Joy & 11.3 & Angry & 3.5 \\
\hline Sad & 2.6 & Fearful* & 0.6 \\
\hline Positive & 8.2 & Neutral & 4.4 \\
\hline Negative & 9.9 & Non-emotion & 59.5 \\
\hline
\end{tabular}

Table 1: The class distribution in Cheng emotion corpus for EClass. (*: ignored)

During our joint training process, the dimension of the word embeddings is 20; the output dimension of the BiLSTM layer used in both the LSTM network and the attention network is 128 ; the output dimension of the linear network is 8 ; the batch size is 32 .

The two models (JMEClass and JMECause) which are learned by our joint approach are compared with several pipeline models which are learned in a pipeline manner (i.e. either for EClass or for ECause) using one of the following state-of-the-art encoders.

- ATT: the attention network in Fig.2 .

- LSTM: the LSTM network in Fig.2.

- ATT+LSTM: an hybrid encoder for emotion classification, which applies $A T T$ to $C u r C L$ and LSTM to PrevCL and FolCL.

- ConvMSMemnet: the encoder proposed by Gui et al. (2017) for emotion cause detection, which applies a convolutional multiple-slot deep memory network to CauseCL.

\subsection{Method Analysis}

Table 2 shows the performances of different emotion classification models, where "Sequence" lists the sequences of input words used by each model and each metric is the average performances of six emotion classes. Moreover, Table 3 lists the detailed performances of each emotion class in Model JMEClass.

\begin{tabular}{|l|l|l|l|l|}
\hline Encoder & Sequence & Prec & Rec & F1 \\
\hline LSTM & CurCL & 65.5 & 53.0 & 58.2 \\
\hline \multirow{2}{*}{ ATT } & CurCL & 67.6 & 56.5 & 61.0 \\
\cline { 2 - 5 } & all & 67.5 & 56.7 & 61.2 \\
\hline ATT+LSTM & all & 67.0 & 57.8 & 61.7 \\
\hline JMEClass & all & $\mathbf{6 7 . 7}$ & $\mathbf{5 8 . 5}$ & $\mathbf{6 2 . 4}$ \\
\hline
\end{tabular}

Table 2: The performances of emotion classification models. (all: PrevCL, CurCL plus FolCL)

\begin{tabular}{|l|l|l|l|}
\hline Class & Prec & Rec & F1 \\
\hline Joy & 85.5 & 83.6 & 84.5 \\
\hline Angry & 62.8 & 45.0 & 52.4 \\
\hline Sad & 72.6 & 72.9 & 72.8 \\
\hline Positive & 63.0 & 54.7 & 58.6 \\
\hline Neutral & 62.5 & 41.2 & 49.7 \\
\hline Negative & 59.9 & 53.9 & 56.7 \\
\hline
\end{tabular}

Table 3: The performances of the six emotions in JMEClass

In Table 2, Model $A T T+C u r C L$ out-performs $L S T M+C u r C L$ by $2.8 \%$ in F-scores, where ATT is a state-of-the-art encoder for emotion classification (Felbo et al., 2017). The significant performance improvement means that ATT can effectively extract affective features in CurCL. In fact, the emotion classification on Chinese microblogs can rely much on emotion keywords occurring in CurCL. E.g. $\sim 50 \%$ emotional instances in our experimental data contains emoticons (e.g. "(6)" in Fig. 1) in CurCL and those emoticons themselves are strong emotion indicators. Secondly, when different kinds of contextual information are incorporated to Model $A T T+C u r C L$, different performance improvements obtain $(0.2 \%$ for $A T T+$ all and $0.7 \%$ for $A T T+L S T M$ in F-scores). This indicates that for the emotion classification, the event-based features extracted by LSTM are more helpful than the affective features extracted by $A T T$, because contexts often provide the cause event of an emotion. E.g. in Fig. 1, the previous clause of " " contains its cause "only I was at home again". Finally, taking the advantage of the event-based features extracted by JMECause, JMEClass out-performs the best pipeline model $(A T T+L S T M)$ by $0.7 \%$ in F-scores. This shows that it is important for the emotion classification to have an encoder which can effectively extract event-based features from contexts.

In Table 3, the performance of a basic emotion (i.e., joy, angry or sad) is often better than the one of a complex emotion (i.e., positive, neutral or negative). However, in Table 1, the data size of a 
basic emotion is often smaller than the one of a complex emotion. This indicates that difference in performance is likely linked to differences in the emotional contents of labels rather than differences in data sizes. E.g. the complex emotion 'negative' (i.e. a collection of complex emotions with negativity, such as 'hate', 'anxious', and so on) is more diverse than the basic emotion 'sad', and this diversity in emotional contents brings more challenges to the detection of this complex emotion. Furthermore, even if both 'sad' and 'angry' are basic emotions and have similar data sizes in our experimental data, it seems much easier to detect 'sad' instances than to detect 'angry' instances. This is maybe because 'angry' is caused by more various events and it is more difficult to capture and utilize those cause events. Thus, it is necessary for the emotion classification to have an encoder which can extract the eventbased information of emotion cause from texts.

\begin{tabular}{|l|l|l|l|l|}
\hline Encoder & Sequence & Prec & Rec & F1 \\
\hline $\begin{array}{l}\text { ConvMS- } \\
\text { Memnet }\end{array}$ & CauseCL & 34.3 & $\mathbf{7 7 . 5}$ & 47.5 \\
\hline ATT & all & 55.4 & 60.9 & 58.0 \\
\hline LSTM & all & $\mathbf{5 5 . 6}$ & 61.3 & 58.3 \\
\hline JMECause & all & 53.1 & 66.7 & $\mathbf{5 9 . 1}$ \\
\hline
\end{tabular}

Table 4: The performances of emotion cause detection models. (all: $E m o K W$, CauseCL plus Context)

Table 4 shows the performances of different emotion cause detection models, where "Sequence" lists the sequences of input words used by each model. In Table 4, JMECause outperforms the best pipeline model (LSTM) by $0.8 \%$ in F-scores. The LSTM encoder is a stateof-the-art approach used for emotion cause detection (Cheng et al., 2017). Furthermore, the performance improvement of JMECause is from the significant increasing in recalls $(5.4 \%)$. This indicates that more emotion causes are correctly detected when the event-based features extracted by Model JMEClass are incorporated. Moreover, among all models, the two models (ATT and LSTM) achieve relatively high precision and relatively low recall, and ConvMS-Memnet obtains the lowest precision and highest recall. This means that both ATT and LSTM suffer from the feature coverage problem because some useful features cannot be extracted through their encoders, and ConvMS-Memn suffers from the feature quality problem maybe because its encoder cannot handle the informal writing style used in Chinese microblogs.

\section{Related Work}

In recent years, intensive studies have explored supervised machine learning approaches using various types of features for different-level emotion classification, such as document level (Alm et al. 2005; Li et al. 2014; Huang et al. 2016), sentence level or short text level (Tokushisa et al. 2008; Bhowmick et al. 2009; Xu et al. 2012; Wen and Wan, 2014; Li et al. 2015; Felbo et al., 2017), and so on. Moreover, since both emotion and sentiment belong to affective feeling, some studies have explored the join learning of sentiment classification and emotion classification (Gao et al., 2013; Wang et al., 2015).

In the other hand, most of previous emotion cause detection studies is clause-based, which examine whether a clause around a given emotion keyword is a cause or not. Moreover, these studies (Chen et al., 2010; Xu et al., 2017; Ghazi et al., 2015; Gui et al., 2017; Cheng et al., 2017) focus on how to extract two kinds of features for supervised model learning: explicit expression patterns (e.g. "to cause", "for"), and implicit features which can reflect the causal relation.

\section{Conclusion}

In this paper, we focus on a joint learning approach to emotion classification and emotion cause detection on Chinese microblogs, and the experiments show such a joint approach is very promising.

\section{Acknowledgments}

This research work was partially supported by four the National Science Foundation of China (No. 61503386).

\section{References}

Cecilia Ovesdotter Alm, Dan Roth and Richard Sproat. 2005. Emotions from Text: Machine Learning for Text-based Emotion Prediction. In Proceedings of Human Language Technology Conference and Conference on Empirical Methods in Natural Language Processing (HLT/EMNLP), pages 579-586.

Plaban Kr. Bhowmick, Anupam Basu, Pabitra Mitra and Abhishek Prasad. 2009. Multi-label Text Clas- 
sification Approach for Sentence Level News Emotion Analysis. In Proceedings of the International Conference on Pattern Recognition and Machine Intelligence, pages 261-266.

Ying Chen, Sophia Yat Mei Lee, Shoushan Li and Chu-Ren Huang. 2010. Emotion cause detection with linguistic constructions. In Proceedings of the 23rd International Conference on Computational Linguistics (Coling 2010), pages 179-187.

Xiyao Cheng, Ying Chen, Bixiao Cheng, Shoushan Li and Guodong Zhou. 2017. An Emotion Cause Corpus for Chinese Microblogs with MultipleUser Structures. In ACM Transactions on Asian and Low-Resource Language Information Processing (TALLIP), Vol. 17, No. 1, Article 6.

Bjarke Felbo, Alan Mislove, Anders Søgaard, Iyad Rahwan and Sune Lehmann. 2017. Using millions of emoji occurrences to learn any-domain representations for detecting. In Proceedings of the 2017 Conference on Empirical Methods in Natural Language Processing, pages 1615-1625.

Wei Gao, Shoushan Li, Sophia Yat Mei Lee, Guodong Zhou and Chu-Ren Huang. 2013. Joint Learning on Sentiment and Emotion Classification. In Proceedings of the 22nd ACM international conference on Information \& Knowledge Management, pages 1505-1508.

Diman Ghazi, Diana Inkpen and Stan Szpakowicz. 2015. Detecting emotion stimuli in emotionbearing sentences. In Proceedings of the 16th International Conference on Computational Linguistics and Intelligent Text Processing, pages 152165 .

Lin Gui, Jiannan $\mathrm{Hu}$, Yulan He, Ruifeng Xu, Qin Lu and Jiachen Du. 2017. A Question Answering Approach to Emotion Cause Extraction. In Proceedings of the 2017 Conference on Empirical Methods in Natural Language Processing, pages 15931602.

Lei Huang, Shoushan Li and Guodong Zhou. 2016. Emotion Corpus Construction on Microblog Text. The series Lecture Notes in Computer Science, Volume 9332, pages 204-212.

Chengxin Li, Huimin Wu and Qin Jin. 2014. Emotion Classification of Chinese Miroblog Text via Fusion of Bow and Evector Feature Representations. In Proceedings of the 3rd CCF Conference on Natural Language Processing \& Chinese Computing, pages 217-228.

Shoushan Li, Lei Huang, Rong Wang and Guodong Zhou. 2015. Sentence-level Emotion Classification with Label and Context Dependence. In Proceedings of the 53rd Annual Meeting of the Association for Computational Linguistics and the 7th Interna- tional Joint Conference on Natural Language Processing, pages 1045-1053.

Ryoko Tokuhisa, Kentaro Inui and Yuji Matsumoto. 2008. Emotion Classification Using Massive Examples Extracted from the Web. In Proceedings of the 22nd International Conference on Computational Linguistics (Coling 2008), pages 881-888.

Jun Xu, Ruifeng Xu, Qin $\mathrm{Lu}$ and Xiaolong Wang. 2012. Coarse-to-fine Sentence-level Emotion Classification based on the Intra-sentence Features and Sentential Context. In Proceedings of the 21st ACM International Conference on Information and Knowledge Management, pages 2455-2458.

Ruifeng $\mathrm{Xu}$, Jiannan $\mathrm{Hu}$, Qin $\mathrm{Lu}$, Dongyin $\mathrm{Wu}$ and Lin Gui. 2017. An Ensemble Approach for Emotion Cause Detection with Event Extraction and Multi-Kernel SVMs. TSINGHUA SCIENCE AND TECHNOLOGY, Volume 22, Number 6.

Rong Wang, Shoushan Li, Guodong Zhou and Hanxiao Shi. 2015. Joint Sentiment and Emotion Classification with Integer Linear Programming. In Liu A., Ishikawa Y., Qian T., Nutanong S., Cheema M. (eds) Database Systems for Advanced Applications, Lecture Notes in Computer Science, Vol 9052.

Shiyang Wen and Xiaojun Wan. 2014. Emotion Classification in Microblog Texts Using Class Sequential Rules. In Proceedings of the Twenty-Eighth AAAI Conference on Artificial Intelligence, pages 187-193. 